\title{
The small GTPase RhoU lays downstream of JAK/STAT signaling and mediates cell migration in multiple myeloma
}

\author{
Sara Canovas Nunes ${ }^{1,2}$, Martina Manzoni $\mathbb{1}^{3,4}$, Marco Pizzi ${ }^{5}$, Elisa Mandato 1,2,6, Marilena Carrino ${ }^{1,2}$, Laura Quotti Tubi ${ }^{1,2}$, \\ Renato Zambello 1,2, Fausto Adami', Andrea Visentin (1)', Gregorio Barilà', Livio Trentin (1) 1,2, Sabrina Manni ², \\ Antonino Neri, ${ }^{3,4}$ Gianpietro Semenzato ${ }^{1,2}$ and Francesco Piazza ${ }^{1,2}$
}

\begin{abstract}
Multiple myeloma is a post-germinal center B-cell neoplasm, characterized by the proliferation of malignant bone marrow plasma cells, whose survival and proliferation is sustained by growth factors and cytokines present in the bone marrow microenvironment. Among them, IL-6 triggers the signal downstream of its receptor, leading to the activation of the JAK/STAT pathway. The atypical GTPase RhoU lays downstream of STAT3 transcription factor and could be responsible for mediating its effects on cytoskeleton dynamics. Here we demonstrate that $\mathrm{RHOU}$ is heterogeneously expressed in primary multiple myeloma cells and significantly modulated with disease progression. At the mRNA level, RHOU expression in myeloma patients correlated with the expression of STAT3 and its targets MIR21 and SOCS3. Also, IL-6 stimulation of human myeloma cell lines up-regulated RHOU through STAT3 activation. On the other hand, RhoU silencing led to a decrease in cell migration with the accumulation of actin stress fibers, together with a decrease in cyclin D2 expression and in cell cycle progression. Furthermore, we found that even though lenalidomide positively regulated RhoU expression leading to higher cell migration rates, it actually led to cell cycle arrest probably through a p21 dependent mechanism. Lenalidomide treatment in combination with RhoU silencing determined a loss of cytoskeletal organization inhibiting cell migration, and a further increase in the percentage of cells in a resting phase. These results unravel a role for RhoU not only in regulating the migratory features of malignant plasma cells, but also in controlling cell cycle progression.
\end{abstract}

\section{Introduction}

Multiple myeloma (MM) is a post-Germinal Center cancer characterized by a multifocal proliferation of clonal, long-lived plasma cells (PCs) within the bone marrow $(\mathrm{BM})^{1}$. This multistep malignancy is preceded by an ageprogressive premalignant condition called monoclonal gammopathy of undetermined significance (MGUS $)^{1-3}$. Some patients pass through a phase called smoldering

\footnotetext{
Correspondence: Francesco Piazza (francesco.piazza@unipd.it)

${ }^{1}$ Department of Medicine, Division of Hematology, University of Padova,

Padova, Italy

${ }^{2}$ Laboratory of Normal and Malignant Hematopoiesis, Venetian Institute of

Molecular Medicine, Padova, Italy

Full list of author information is available at the end of the article
}

myeloma (sMM), in which some of the diagnostic criteria for MM are met but there are no clinical manifestations ${ }^{2}$. In early stages, MM cells like normal long-lived PCs are highly dependent on the $\mathrm{BM}$ microenvironment that activates multiple pathways, protecting these cells from apoptosis ${ }^{4}$. IL-6, primarily produced by BM stromal cells (BMSCs), is the best characterized MM growth factor and is highly responsible for cell homing, seeding, proliferation, and survival through the activation of the JAK/STAT pathway ${ }^{2,4}$.

The Rho family of small guanosine triphosphatases (GTPases) forms part of the Ras super-family. These GTPases share a common biochemical mechanism, acting as molecular switches to transduce the signal downstream 
to their effectors ${ }^{5}$. To note, the Ras family has been proven to profoundly influence cell growth and activating mutations of Ras are associated with cancer ${ }^{6}$. In contrast, Rho GTPases are hardly ever found mutated but often display altered activity in malignant cells when compared to healthy counterparts ${ }^{7}$. Rho GTPases are potent regulators of cytoskeleton dynamics and of the actin filament system, thereby affecting the morphologic and migratory properties of cells ${ }^{8}$. Due to their important roles in controlling these cellular processes, deregulated Rho GTPases could be at the basis of many tumorigenic events.

The RhoU/V sub-family is particularly interesting due to its unique domain organization. Both members of this family, RhoU and RhoV, have an N-terminal proline-rich domain that is not present in any other Rho GTPase and that enables them to permanently bind to their effectors $^{7,9}$. RhoU has no detectable GTPase activity but its very high intrinsic guanine nucleotide exchange activity is likely to ensure that the protein is predominantly in the GTP-loaded conformation ${ }^{10}$. It is encoded by the RHOU gene at 1q42.13 and its expression is mainly controlled at the RNA level downstream of Wnt-1 and STAT3 activation and it might mediate the effects of these signaling pathways in regulating cell morphology, cytoskeletal organization, and proliferation ${ }^{11}$. Also, different levels of this GTPase might lead to diverse outcomes in cell morphology. It is known that during epithelialmesenchymal transition of neural crest cells, high levels of RhoU influence cell polarity and migration while low levels are required for cell adhesion ${ }^{12}$. While typical Rho proteins, such as Cdc42 and Rac1 that share significant sequence homology with RhoU, have an established role in cancer, very little is known about RhoU in tumorigenesis in particular in hematologic malignancies ${ }^{7}$. Since RhoU can alter cell adhesion, actin dynamics, and cell motility, we aimed at testing if this protein could mediate these cellular features in myeloma cells and if changes in its expression, and thus activity, might lead to BM niches remodeling.

\section{Materials and methods}

\section{Patient samples and healthy donors}

PCs were purified from BM samples using CD138 immunomagnetic microbeads (MidiMACS system, Miltenyi Biotec, Auburn, CA) and the purity of the positively selected PCs was $>90 \%$ in all cases. Gene expression profiles (GEP) were investigated in a panel of 268 patients included in two different datasets and representative of all the major forms of PC dyscrasia: a proprietary dataset at NCBI Gene Expression Omnibus repository (accession \#GSE66293) previously profiled by us (4 normal controls and $129 \mathrm{MM}$ patients) ${ }^{13,14}$; and a publicly available data set including five normal controls, 20 MGUS, 33 SMM, and 41 MM patients (accession \#GSE47552) ${ }^{15}$. The cohort consists of newly-diagnosed patients. The proprietary 129 MM tumors (accession \#GSE66293) employed for the study were representative of the major molecular characteristics of the disease and stratified according the TC classification ${ }^{16,17}$. Deletions of $17 \mathrm{p} 13$, 13q14 and gain of 1q were also evaluated by $\mathrm{FISH}^{18}$. Procedures followed the rules indicated in the declaration of Helsinki. The internal Institutional Board approved the use of human material.

\section{Cell lines}

INA-6 cell line was a kind gift from Dr. Martin Gramatzki (University of Kiel, Germany). U266, H929, RPMI8226, and HS-5 were purchased from ATCC-LGC Standards (Milan, Italy). SaMMi cell line was generated in our laboratory ${ }^{19}$. Cell cultures were kept under controlled atmosphere at $37^{\circ} \mathrm{C}$ in the presence of $5 \% \mathrm{CO}_{2}$ and were periodically checked for Mycoplasma contamination.

\section{SiRNA transfection}

U266 cells were transfected with Amaxa Nucleofector ${ }^{\circ}$ Kit C (Lonza, USA) and $100 \mathrm{pmol}$ of RhoU siRNA (Dharmacon ON-TARGETplus RHOU siRNA, Euroclone, Italy) or $100 \mathrm{pmol}$ of Scrambled (Dharmacon ONTARGETplus non-targeting siRNA, Euroclone, Italy) according to the manufacturer's instruction.

\section{Chemicals}

$2 \times 10^{6}$ cells were cultured in $2 \mathrm{ml}$ of favorite medium, stimulated with $10 \mathrm{ng}$ of IL-6 (ImmunoTools, Germany) and samples were collected at different time points. Cell lines that are usually cultured in medium supplemented with IL-6 were starved from this cytokine for $12 \mathrm{~h}$ previous to stimulus.

Stattic (Selleckchem, USA) was employed for $5 \mathrm{~h}$ at different doses to inhibit STAT3 and then IL- 6 was added or not to the cells for $1 \mathrm{~h}$. Cells were starved of IL- 6 only during the $6 \mathrm{~h}$ of treatment.

Lenalidomide (Selleckchem, USA) was used at different doses for 4 or $24 \mathrm{~h}$.

\section{Transwell migration assay}

$5 \mu$ mTranswell ${ }^{\circ}$ Permeable Supports on 24 well plates (Corning, USA) were used according to manufacturer instructions. Six hundred microliter non-supplemented medium $+0.1 \%$ BSA were added to the bottom of multiwell plate and $50 \mu \mathrm{L}$ of the same mix were added on top of the transwell insert. $4 \times 10^{5}$ cells were washed with HBSS buffer and then resuspended in $50 \mu \mathrm{L}$ of recommended medium $+0.1 \%$ BSA and added to the top well insert. Plates were incubated for $20 \mathrm{~min}$ and then IL-6 stimulus (10 ng) or MSC culture medium (from 24 h MSC culture) was carefully added to the bottom well. IL-6 or MSC 


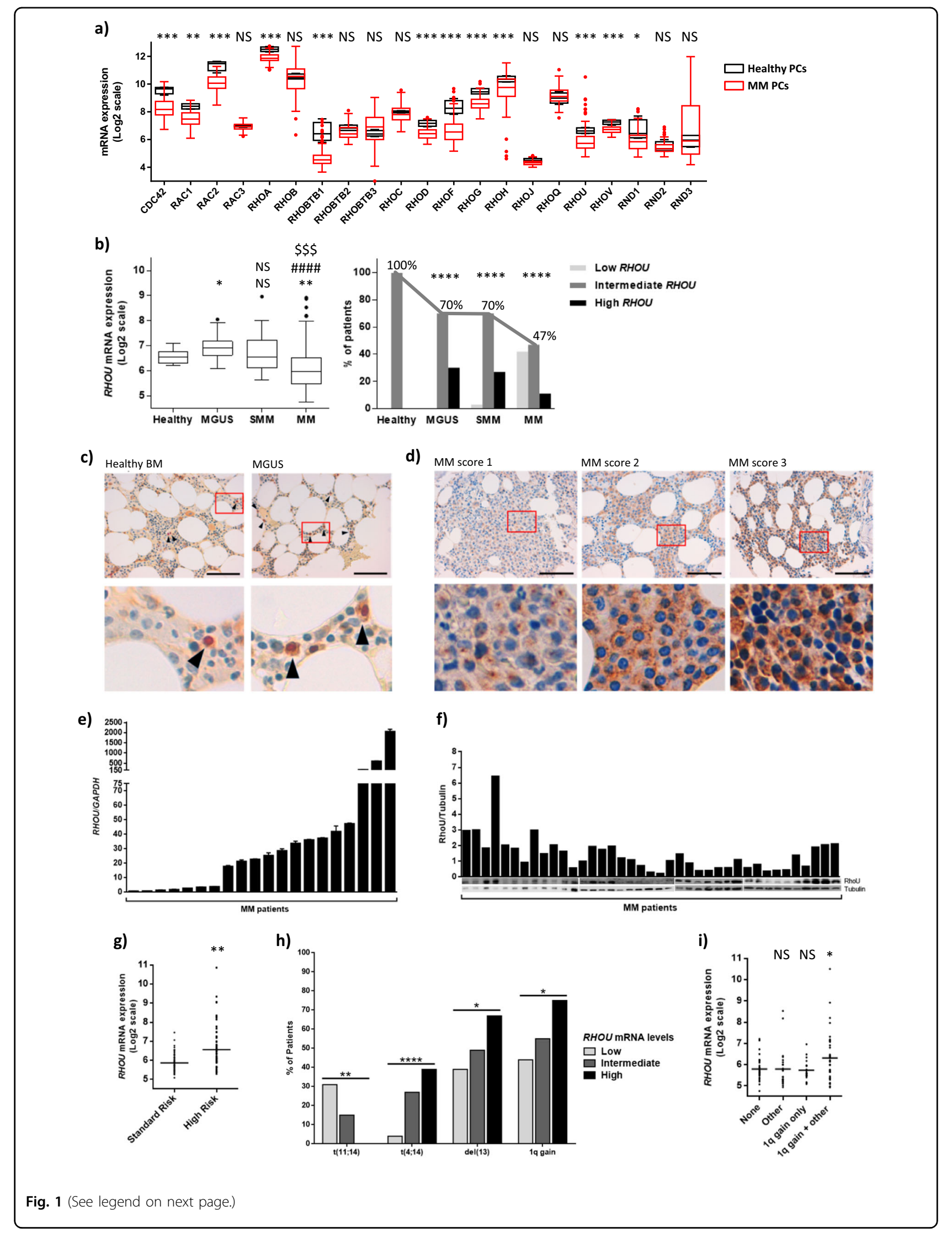


Fig. 1 RhoU is heterogeneously expressed in MM. a Rho GTPase family members' expression in healthy and MM PCs. Box plot showing mean \pm SD of healthy PCs from four donors (red) and mean \pm SD of MM PCs from 129 MM patients at diagnosis (black). In the $x$ axis are all the members of the Rho GTPase family in alphabetical order. Student's $t$ test ${ }^{*} p<0.05 ;{ }^{* *} p<0.01 ;{ }^{* *} p<0.001$ when compared to healthy PCs. b left: $R H O U$ expression in the different steps of MM progression. Box plot showing mean \pm SD of healthy PCs from nine donors, MGUS PCs from 20 patients, sMM PCs from 33 patients and MM PCs from 170 patients at diagnosis. Student's $t$ test: NS non significant; ${ }^{*} p<0.05 ;{ }^{* *} p<0.01 ;{ }^{* * *} p<0.001 ;{ }^{* * * *} p<0.0001$ ( ${ }^{*}$ compared to healthy; ${ }^{*}$ compared to MGUS; ${ }^{\$}$ compared to SMM). right: Bar chart showing the percentage of patients for each step of the disease that fall in the diverse groups of RHOU expression. Patients were divided in three groups with low (<mean $-2 \mathrm{SD}$ of healthy controls), intermediate, and high (>mean +2 SD of healthy controls) RHOU expression. Chi square test of patients' distribution: ${ }^{* * *} p<0.0001$ when compared to healthy. $\mathbf{c}$ RhoU expression in BM biopsies of a healthy donor and a representative MGUS patient. PCs in the samples are indicated with black arrows. Nucleuses of PCs are positive for IRF4 staining and are therefore red. $\mathbf{d} I \mathrm{HC}$ staining showing low (MM score 1), intermediate (MM score 2) and high (MM score 3) positivity for RhoU staining in BM biopsies from representative MM patients. $\mathbf{c}$ and $\mathbf{d}$ Original magnification x60; inset with x5 digital zoom; scale bar $=100 \mu \mathrm{m}$. e Bar charts showing qRT-PCR RHOU/GAPDH ratio values in PCs purified from 20 MM patient's biopsies. Mean \pm SD of technical triplicates is shown for each samples. f Immunoblot and densitometry of RhoU/Tubulin in PC lysates from 39 MM patient's biopsies. $\mathbf{g}$ Aligned dot plot with RHOU expression in patients that fall in the standard risk (TC1 and TC2) and high risk (TC3, TC4, and TC5) groups. Mean expression in each group is marked with a bar. $p$ value was calculated by Student's $t$ test. $\mathbf{h}$ Bar chart representing the percentage of patients from each of the three groups with low (<mean-2 SD of healthy controls), intermediate, and high ( $>$ mean +2 SD of healthy controls) RHOU expression that exhibit a particular genetic alteration. Student's $t$ test for trend ${ }^{*} p<0.05 ;{ }^{* *} p<0.01$; ${ }^{* * *} p<0.0001$. i Aligned dot plot with RHOU expression in patients that don't present any unfavorable cytogenetic alterations (none), that present unfavorable cytogenetic alterations but not 1q gain (other), that present only $1 q$ gain, and that present 1q gain combined with other unfavorable cytogenetic alterations. Student's $t$ test: NS non significant; ${ }^{*} p<0.05$

culture medium was not added to control wells. Plates were incubator for $6 \mathrm{~h}$ before collection.

\section{Immunohistochemistry (IHC)}

IHC was performed on $4 \mu \mathrm{m}$-thick formalin-fixed, paraffin-embedded sections of BM biopsies of 1 healthy and 8 MGUS using anti-RhoU (HPA049592, SigmaAldrich, USA) and anti-IRF4 (HPA002038, SigmaAldrich, USA) monoclonal primary antibodies. Fifteenmiilimeter biopsies were stained with anti-RhoU and zones with a clearly abundant plasmacytosis were used in evaluating RhoU positivity. All sections were processed using the sensitive Bond Polymer Refine Detection kit, a biotin-free, polymeric horseradish peroxidase-linker antibody conjugate system, in an automated immunostainer (Bond maX, Menarini, Italy). Appropriate positive and negative controls were run concurrently. RhoU immunostain was semiquantitatively scored in a fourtiered scale, as follows: score $0=$ negative staining; score $1=$ weak positivity staining; score $2=$ moderate positivity staining; score $3=$ strong positive staining. Immunohistochemical reactions were independently scored by two investigators (agreement $k>0.8$ ).

\section{Immunofluorescence (IF)}

$5 \times 10^{4}$ cells were seeded on polylysine-coated glass slides and incubated at $37^{\circ} \mathrm{C}$ for $1 \mathrm{~h}$ to let them adhere to the polylysine, fixed with formaldehyde $3.7 \%$, permeabilized with Triton $0.1 \%$ and blocked with BSA 3\%. Samples were then stained with Phalloidin Alexa Fluor 594 (Invitrogen, USA) for 30', mounted in Vectashield mounting medium with DAPI (4',6-diamidino-2-phenylindole) (Vector Laboratories, USA) and analyzed using ZEISS LSM700 confocal microscope with $63 \times$ magnification objective. Images were analyzed with ImageJ software.

\section{Results}

\section{Rho GTPases display altered expression in MM PCs}

We have assessed the expression of the Rho GTPase family members in PCs from BM biopsies of MM patients and in normal BM PCs from healthy donors, using the gene expression profiling (GEP) of 129 MM patient samples at diagnosis and four healthy controls included in the proprietary GEO data set GSE66293. We found that Rho GTPase family members are differently expressed in MM PCs when compared to healthy PCs (Fig. 1a). More precisely, 12 (CDC42, RAC1, RAC2, RHOA, RHOBTB1, RHOD, RHOF, RHOG, RHOH, RHOU, RHOV, and RND1) out of the 21 GTPases analyzed, were expressed at significantly lower levels in MM when compared to healthy controls $(p<0.05)$.

\section{RhoU is heterogeneously expressed in different steps of MM progression}

Next, we combined the data available in two GEP data sets (\#GSE66293 and \#GSE47552) as previously described $^{13}$ and found that $R H O U$ expression is significantly and negatively modulated with disease progression, being over-expressed in BM PCs from MGUS patients and down-modulated in BM PCs from most MM patients (Fig. $1 \mathrm{~b}$ left panel). However, we found a large heterogeneity in RHOU expression, with several patients showing high transcript levels even in late stages. Therefore, we divided patients in three groups according to low $(<$ mean -2 SD of healthy controls), intermediate $(=$ mean $\pm 2 \mathrm{SD}$ of healthy controls), and high (>mean $+2 \mathrm{SD}$ of healthy controls) expression. Along with disease progression there was an evident decrease in the percentage of patients that fell in the group with intermediate $R H O U$ expression (Fig. $1 \mathrm{~b}$ right panel) and an increase in heterogeneity. In MM in particular, there were three distinct groups of patients: 


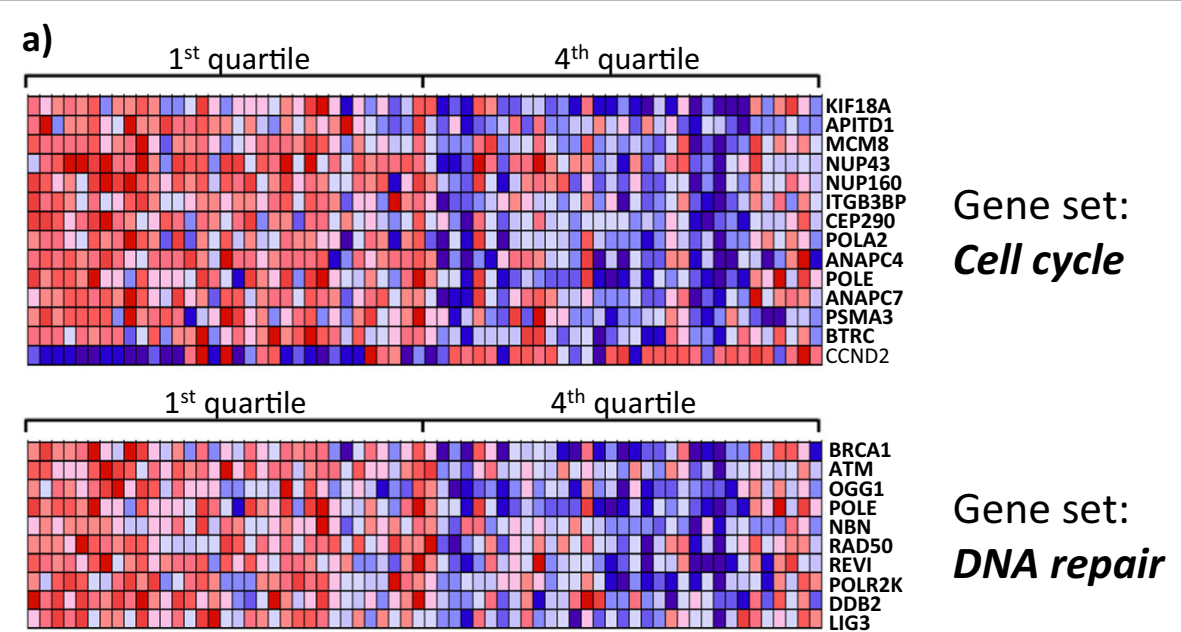

b)

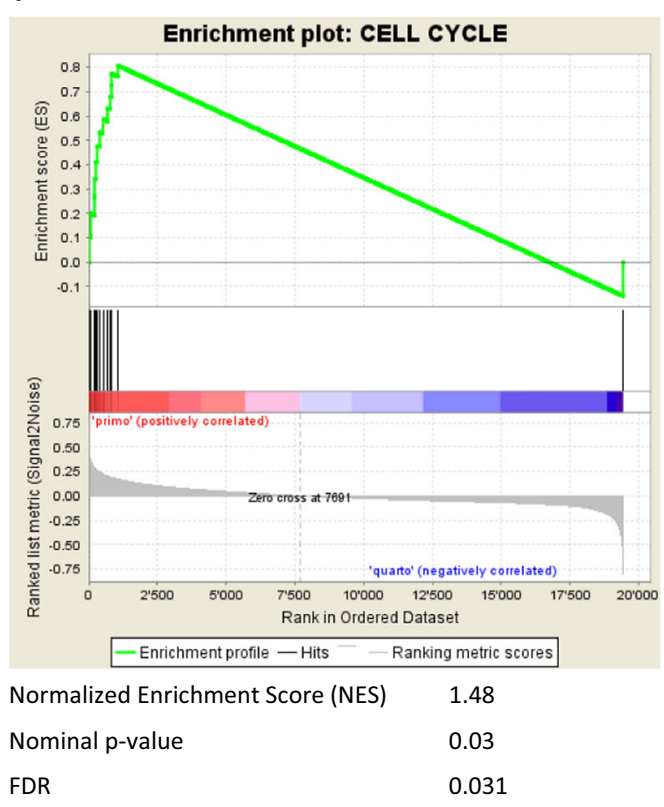

d)

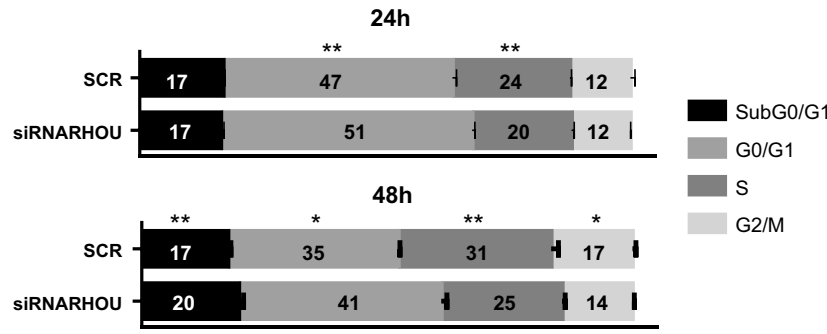

c)

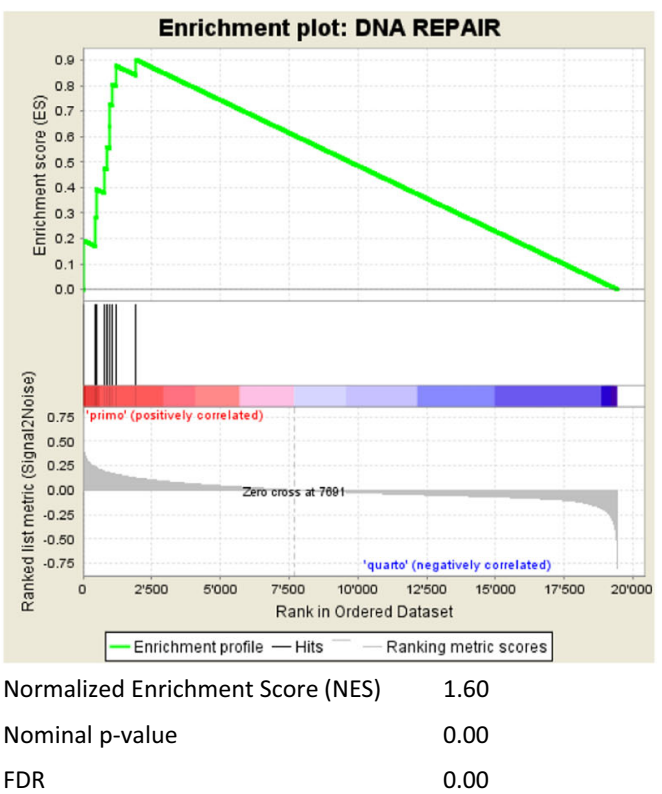

e)

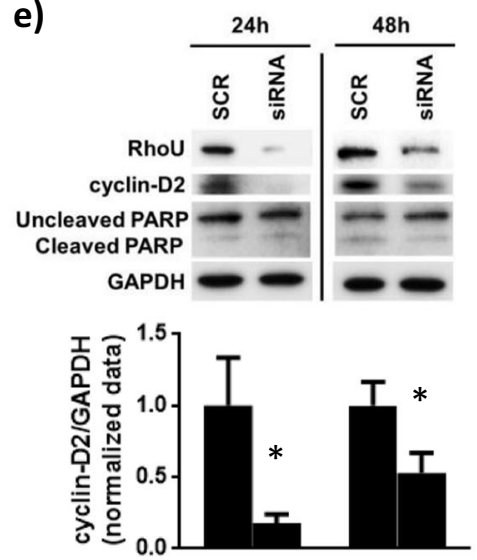

Fig. 2 (See legend on next page.) 
Fig. 2 Patients with high and low RHOU expression have different gene expression profiles. a Heat map of genes contributing to the core enrichment in the "Cell Cycle" and "DNA Repair" gene sets detected by GSEA analyzing significantly up-regulated genes ( $p$-value $\leq 0.05)$ in RHOU quartile I versus IV (indicated in bold). Enrichment plots for b "Cell Cycle" and c "DNA Repair" gene sets. The green curves show the enrichment score and reflect the degree to which each gene (black vertical lines) is represented the bottom of the ranked gene list. $\mathbf{d}$ Cell cycle analysis based on PI staining of SCR and siRNA RHOU cells 24 and $48 \mathrm{~h}$ after transfection. Data represents mean \pm SD of three independent experiments. Student's $t$ test: ${ }^{*} p<0.05$; ${ }^{* *} p<0.01$. e Representative immunoblots from six samples from two independent experiments of RhoU and cyclin D2 expression 24 and $48 \mathrm{~h}$ after siRNA transfection, and densitometry of the mean expression of cyclin D2/GAPDH \pm SD

$42 \%$ with low, $47 \%$ with intermediate, and $11 \%$ with high RHOU expression.

To further examine RhoU expression we performed IHC analysis in an independent panel of patients including 8 MGUS and 15 active MM. All MGUS patients' PCs showed a high intensity cytoplasmic staining for RhoU when compared to healthy PCs (Fig. 1c). Also, PCs from all MM patients showed positivity for RhoU staining and were given a score based on the intensity. More precisely $20 \%$ showed weak (score 1), 40\% moderate (score 2), and $40 \%$ high RhoU (score 3) positivity, consistent with the heterogeneous levels of RHOU mRNA described above (Fig. 1d). Cells with different scores seem to also have a diverse localization of RhoU: in MM cells with score 1 RhoU had a dot-like localization in proximity of the nucleus, which could represent the actin microtubuleorganizing center, whereas cells that scored 2 or 3 showed a granular cytoplasmic and membrane localization. The heterogeneous levels of RhoU in MM patients were also validated by qRT-PCR (Fig. 1e) and Western Blot (Fig. 1f).

Next, we evaluated $R H O U$ levels according to the major molecular groups of MM patients based on the TC classification as previously described ${ }^{17}$. Patients were further divided in two groups: standard risk patients that fall into TC1 and TC2 groups $(n=64)$ and characterized by $t$ $(11,14)$ (TC1) and hyperdiploidy (TC2) leading to cyclin D1 expression, and high risk patients that comprise TC3, TC4, and TC 5 groups $(n=65)$ characterized by cyclin D2 expression alone (TC3) and presence of $t(4 ; 14)$ (TC4) or MAF translocations (TC5). The results show that patients in the high risk group have significantly higher levels of RHOU expression (Fig. 1g), compared to TC1/TC2 patients. Furthermore, patients with high $\mathrm{RHOU}$ expression have a higher frequency of $1 \mathrm{q}$ gain, $\operatorname{del}(13)$, and $\mathrm{t}$ $(4 ; 14)$ (Fig. 1h). Finally, since $R H O U$ is located at 1q42 we have divided the patients in four groups, without unfavorable cytogenetic alterations (none), with unfavorable cytogenetic alterations but without 1q gain (other), with 1q gain only, and with 1q gain plus unfavorable cytogenetic alterations, discovering that $1 \mathrm{q}$ gain alone is not enough to cause a significant increase in $R H O U$ expression (Fig. 1i).

To better understand if $R H O U$ expression could correlate with patient survival we have used two datasets: one with expression in untreated patients and overall survival
Table 1 Gene set "Cell Cycle" was detected by GSEA as significantly up-regulated in RHOU quartile I versus IV

GSEA results summary

\begin{tabular}{ll}
\hline Geneset & Cell cycle \\
Genes & ITGB3BP, NUP160, BTRC, POLE, ANAPC4, \\
& $\begin{array}{l}\text { KIF18A, POLA2, MCM8, APITD1, CCND2, } \\
\text { PSMA3, CEP290, ANAPC7, NUP43 }\end{array}$ \\
Normalized Enrichment & 1.48 \\
Score (NES) & \\
Nominal $p$-value & 0.03 \\
FDR & 0.031 \\
\hline
\end{tabular}

ITGB3BP overexpression induces apoptosis in cancer cells ${ }^{24}$; NUP160, NUP43, KIF18A, BTRC, APITD1, and CEP290 are required for correct mitosis, centrosome dynamics, and chromosome alignment ${ }^{25-30}$; POLE, POLA2, and MCM8 have extremely important roles in DNA replication and genome stability ${ }^{31-33}$; ANAPC4 and ANAPC7 have emerging roles in differentiation control, genomic stability and tumor suppression ${ }^{34}$; CCND2 enhances cell cycle progression and MM proliferation $^{35}$ and PSMA3 is part of the proteasome complex and essential for protein degradation during cell cycle progression ${ }^{36}$.

Table 2 Gene set "DNA Repair” was detected by GSEA as significantly up-regulated in RHOU quartile I versus IV

GSEA results summary

\begin{tabular}{ll}
\hline Geneset & DNA repair \\
Genes & NBN, REV1, POLR2K, POLE, DDB2, LIG3, \\
& OGG1, ATM, RAD50, BRCA1 \\
Normalized Enrichment & 1.60 \\
Score (NES) & \\
Nominal $p$-value & 0.00 \\
FDR & 0.00 \\
\hline
\end{tabular}

These genes are extremely important since they encode for proteins essential in a number of cellular pathways that maintain genomic stability, including DNA damage-induced cell cycle checkpoint activation, DNA damage repair, protein ubiquitination, chromatin remodeling, as well as transcriptional regulation and apoptosis $^{37-44}$

after Total Therapy 2 (TT2) and Total Therapy 3 (TT3) regimens (\#GSE2658) ${ }^{20-22}$, and another with expression after several lines of therapy and overall survival after Total Therapy 6 (TT6) (\#GSE57317) ${ }^{23}$. We have divided the patients from each dataset in RHOU expression 


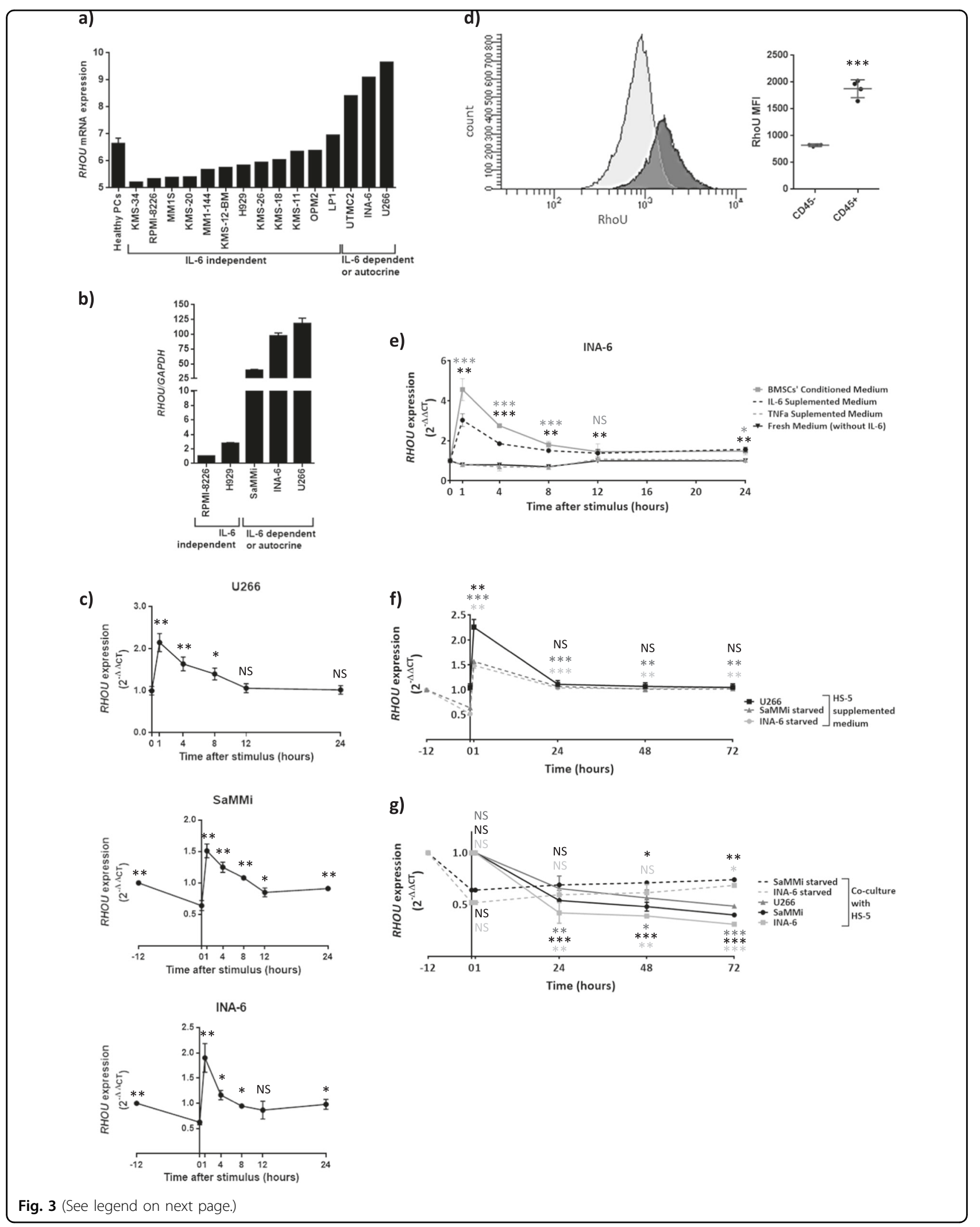


Fig. 3 RHOU expression in MM cell lines relies on IL-6 stimulus. a Bar chart showing RHOU expression assessed by GEP (\#GSE66293) in MM cell lines as compared to healthy PCs. $\mathbf{b}$ Bar chart showing RHOU expression in MM cell lines assessed by RT-PCR. Data represents normalized mean \pm SD of three independent samples of each cell line. c Time-course of RHOU expression after IL-6 stimulus showing a significant increase in $R H O U$ expression as early as one hour after stimulus; time points before zero correspond to IL-6 starvation period. $\mathbf{d}$ Left: representative dot plot showing intracytoplasmic RhoU staining in the CD45+ and CD45- populations of U266 cell line. Right: mean \pm SD of four samples mean fluorescence intensity of intracytoplasmic RhoU staining in the CD45+ and CD45- populations of U266 cell line. e Time-course of RHOU expression after the addition of IL-6, TNF-a, BMSCs' conditioned medium or fresh unsupplemented medium. $\mathrm{f}$ Time-course showing the changes in RHOU expression after the addition of HS-5 supplemented medium of starved and not starved cell lines. $\mathbf{g}$ Time-course showing the changes in RHOU expression after co-culture with HS-5 of starved and not starved cell lines. $\mathbf{c}, \mathbf{e}, \mathbf{f}$, and $\mathbf{g}$ Data represents mean \pm SD of six samples from at least two independent experiments normalized over mean expression at time zero. Student's $t$ test: NS non significant; ${ }^{*} p<0.05 ;{ }^{* *} p<0.01 ;{ }^{* * *} p<0.001$, when compared to time zero of untreated

quartiles and compared the survival curves of the first and fourth quartiles. In TT2 or TT3 regimens there is a $50 \%$ higher risk of death (hazard ratio $=1.50$ ) for the patients in the 4th quartile when compared to patients in the I quartile (Supplementary Figure S1a and b). In TT6 regimen the risk of death increases to $612 \%$ (hazard ratio $=$ 7.12) for patients in the IV quartile when compared to patients in the I quartile (Supplementary Figure S1c and d). These data suggest a positive correlation between high RHOU levels and worse prognosis also after antimyeloma therapies. Whether or not its association with poor outcomes can be linked to the combination of $1 \mathrm{q}$ gain and other unfavorable cytogenetic alterations remains to be investigated.

\section{Patients with high and low RHOU expression have a different GEP}

To gain insights into the role of $R H O U$ in $\mathrm{MM}$, we focused on our proprietary MM group (\#GSE66293) due to the absence of any bias related to sample molecular characteristics, and looked for the gene expression signature associated with $R H O U$ expression. Specifically, we ranked patients in four classes based on RHOU expression level. Next, by comparing the lowest (I quartile) with the highest (IV quartile) $R H O U$ expressing patients, we found 557 genes differentially expressed between the two groups that appeared to be gradually modulated in the four quartiles (Supplementary Table S1). Functional enrichment analysis of the 557 deregulated genes using DAVID Bioinformatics Resources 6.8 evidenced 12 significantly annotation clusters $(E S>1.3)$ and six significantly enriched pathways, among which cell cycle and DNA repair (Supplementary Table S2). In addition, to confirm the correlation between $R H O U$ and these pathways, we used the Gene Set Enrichment Analysis (GSEA) to identify a priori defined sets of genes showing concordant modulation between patients with low and high RHOU expression level. Notably, the cell cycle control and DNA repair gene sets were found highly enriched in MM group expressing lower (I quartile) RHOU levels (Fig. 2a-c).

RHOU clustered with 14 genes that regulate the cell cycle and the mitotic process (Table 1). CCND2 was the only gene in the list that positively correlated with $R H O U$ (Pearson's $r=0.402 ; p$-value $=7.193 \mathrm{e}-15$ ) suggesting that malignant $\mathrm{PCs}$ with high $\mathrm{RHOU}$ expression have increased levels of cyclin D2 and a higher replication rate. All the other genes responsible for cell cycle control and DNA damage response had a negative correlation with RHOU expression (Fig. 2b). $R H O U$ also clustered with 10 DNA damage repair genes in the "DNA Repair" pathway (Table 2). All the ten genes in the list negatively correlate with $R H O U$ expression (Fig. 2c).

Strikingly, in U266 cell line, the inhibition of RhoU by siRNA resulted at $24 \mathrm{~h}$ in a slight but significant accumulation of cells in the G0/G1 phase and a decrease in replicating $\mathrm{S}$ phase cells (Fig. $2 \mathrm{~d}$ ). The results were more evident at $48 \mathrm{~h}$ with an increase in both sub G0/G1 and G0/G1 phases and a decrease in both S and G2/M phases. Since G1 to $\mathrm{S}$ phase transition is tightly regulated by the expression of cyclin D2, we have analyzed this protein's expression after RhoU silencing and confirmed a decrease in its levels as early as $24 \mathrm{~h}$ after transfection; The decrease in RhoU protein levels at these time points was confirmed by western blotting (Fig. 2e).

\section{RHOU is overexpressed in IL-6-dependent MM cell lines and is driven by the activation of STAT3}

To better understand $R H O U$ regulation in MM we have investigated its expression on the GEP data (\#GSE66293) and on five MM cell lines available in our laboratory, two IL-6 dependent (INA-6 and SaMMi), one that autocrinally produces IL-6 (U266) and two IL-6 independent (H929 and RPMI-8226). GEP data relative to MM cell lines shows that only INA-6, U266 and UTCM2 (a cell line that also autocrinally produces IL-6) had high $R H O U$ expression levels (Fig. 3a). We have also investigated RHOU expression by qRT-PCR confirming that RPMI-8226 and H929 expressed RHOU at very low levels, while IL-6 dependent/autocrine cell lines over-expressed it (Fig. 3b). To determine whether IL-6 stimulus could lead to an up-regulation of $R H O U$ expression cells were deprived from this cytokine and subsequently stimulated. Samples were collected at different time points (Fig. 3c). All three IL-6-dependent cell lines up-regulated $R H O U$ as early as 


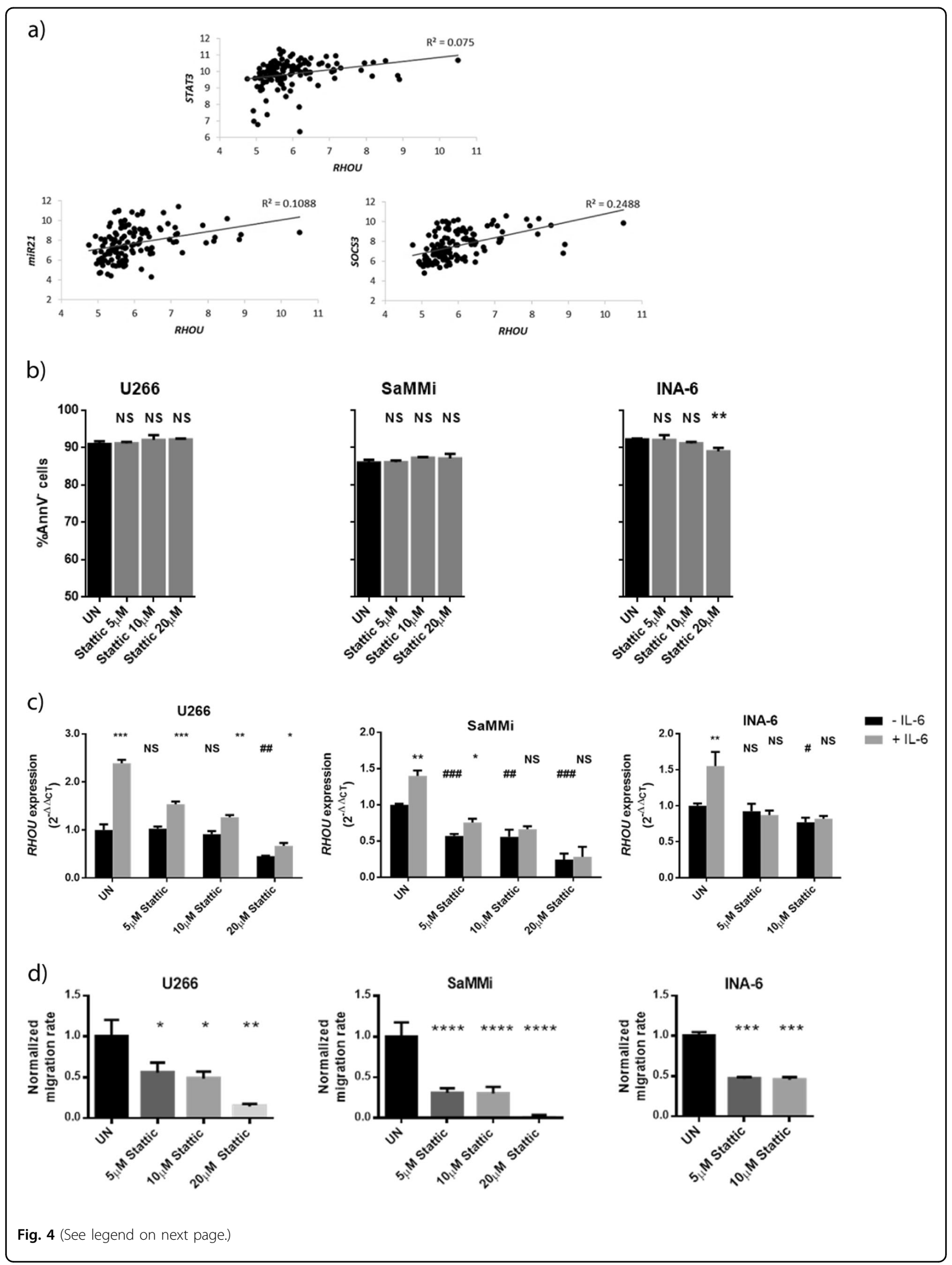


Fig. 4 In MM cell lines RHOU expression lays downstream of STAT3 activation. a Correlation between RHOU expression and STAT3 expression (Pearson's $r=0.2739 ; p$-value $=0.0017$ ) or MIR21 expression (Pearson's $r=0.3299 ; p$-value $=0.0001$ ) or SOCS3 expression (Pearson's $r=0.4988 ; p$-value $=1.786 \mathrm{e}-09$ ). $\mathbf{b}$ Bar charts showing the percentage of live cells (AnnV ${ }^{-}$) after treatment with different doses of Stattic. $\mathbf{c}$ Bar charts showing RHOU expression after STAT3 inhibition before and after IL-6 stimulus. Black bars represent RHOU expression after $6 \mathrm{~h}$ of culture with different concentrations of stattic. Grey bars show RHOU expression after $5 \mathrm{~h}$ of stattic treatment $+1 \mathrm{~h}$ of combined treatment with IL- 6 stimulus. ${ }^{*}$ Compared with same treatment conditions without IL-6 stimulus, " Compared to UN. d Bar charts exhibiting normalized mean cell count \pm SD of four samples from at least two independent experiments, after treatment with increasing concentrations of stattic, accessed by migration assay with IL-6, normalized over mean migration of UN. b, $\mathbf{c}$, and $\mathbf{d}$ UN untreated; student's $t$ test: NS non significant; ${ }^{*} p<0.05 ;{ }^{* *} p<0.01 ;{ }^{* * *} p<0.001$; ${ }^{* * * *} p<0.0001$

$1 \mathrm{~h}$ after stimulus. In detail, $1 \mathrm{~h}$ after the addition of IL-6, there was an increase in $\mathrm{RHOU}$ expression equal to 1.2 times-fold in U266, 1.7 times-fold in SaMMi, and 2.4 times-fold in INA-6. U266 might display the smallest changes due to the fact that this cell line cannot be starved from IL-6 since it autocrinally produces it.

Since CD $45^{+}$myeloma cells have increased JAK/STAT activation $^{45,46}$, we have also studied the $\mathrm{CD} 45^{+}$and CD45 ${ }^{-}$U266 cell populations to further examine RhoU expression in this context. Intracytoplasmic RhoU staining showed a mean fluorescence intensity (MFI) of 822 and of 1878 in the CD45 $5^{-}$and $\mathrm{CD} 45^{+}$cell populations, respectively (Fig. $3 \mathrm{~d}$ ).

To verify if this effect was due to the specific activation of the IL6R/STAT3 pathway, we stimulated cells also with TNF- $\alpha$, conditioned medium from BMSCs' culture, or unsupplemented fresh medium (Fig. 3e). The dynamics of RHOU expression after the addition of BMSCs' conditioned medium or IL-6 stimulus were similar, while TNF$\alpha$ did not cause any significant changes. Adding derived media from HS-5 stromal cell line showed the same results as IL-6 stimulus (Fig. 3f) and a really slow increase in $R H O U$ expression was obtained by co-culturing IL6 starved cell lines with HS-5 cells (Fig. 3g). However, the co-culture on HS-5 cells of non-starved cells, with high levels of RhoU, resulted in an actual decrease in $R H O U$ expression (Fig. 3g). These results may support the hypothesis of a contact dependent down-modulation of RHOU after cell adhesion, as previously observed in neural crest cells ${ }^{47}$.

Interestingly, looking at GEP data from MM patients we found a weak but significant positive correlation between RHOU and STAT3 expression. The analysis of STAT3 targets MIR21 and SOCS3 unraveled however a stronger positive correlation with the expression of $R H O U$ (Fig. 4a).

Next, to verify that STAT3 was the principal transcriptional regulator of $R H O U$ downstream of the IL-6R, we inhibited STAT3 with stattic, a chemical inhibitor that blocks STAT3 phosphorylation, dimerization, and nuclear transition by specifically binding to its $\mathrm{SH} 2$ domain ${ }^{48}$. Based on annexin $\mathrm{V}$ staining, a $6 \mathrm{~h}$ treatment with this drug resulted in a slight but significant increase in cell death only at the highest doses for INA-6 cell line (Fig. 4b). Upon non-toxic doses of stattic treatment we observed a dose-dependent decrease in RHOU expression, while cells were less, if not able, to upregulate the GTPase in response to IL-6 stimulus (Fig. 4c). Stattic treatment also led to a dose dependent decrease in cell migration that could rely on $R H O U$ expression (Fig. 4d).

\section{Lenalidomide treatment increases MM cell migration that is overturned by RhoU inhibition}

To better assess the effects of RhoU in MM cells we have silenced it by transfecting cells with specific siRNA particles. Scrambled non-targeting siRNA (SCR) was used as control. RhoU silencing led to a clear decrease in JNK activating phosphorylation, but did not seem to have an effect on cell death nor on STAT3 activation (Fig. 5a left panel). JNK1 is a target of RhoU, important for the development of filopodia ${ }^{11}$. Therefore, an impairment in its activation could explain the decreased migration capability of RhoU-targeting siRNA transfected cells (Fig. 5a right panel). Furthermore, since IMIDs were shown to be able to regulate the activation of classical Rho proteins in human monocytes ${ }^{49}$, we aimed at studying the effects of lenalidomide on RhoU expression. Immunoblot analysis showed an increase in STAT3 activation after treatment with lenalidomide (Fig. 5b) without a clear effect in viability in U266 (Fig. 5c) and in INA-6 (Supplementary Figure S2a) cell lines. We also observed an increase in RhoU expression and in JNK activating phosphorylation (Fig. 5b and Supplementary Figure S2a) that could result in increased cell mobility. When combining RhoU inhibition with lenalidomide we observed that JNK phosphorylation remained unchanged instead of increasing as happened in UN and SCR conditions (Fig. 5d). When comparing siRNA and SCR transfected cells we observed a significant decrease in migration (Fig. 5e and f). RhoU is thus essential for JNK activation and MM cell migration. Treatment with lenalidomide, that consequently determined higher RhoU and active JNK levels, resulted in a boost in cell migration (Fig. 5e and f; and Supplementary Figure S2b and c). RhoU silencing abolished lenalidomide induced migration (Fig. 5e and f).

We further studied cytoskeleton changes and alterations in cell adhesion by employing IF techniques. Although all cells were able to adhere to polylysine coated glass, the 


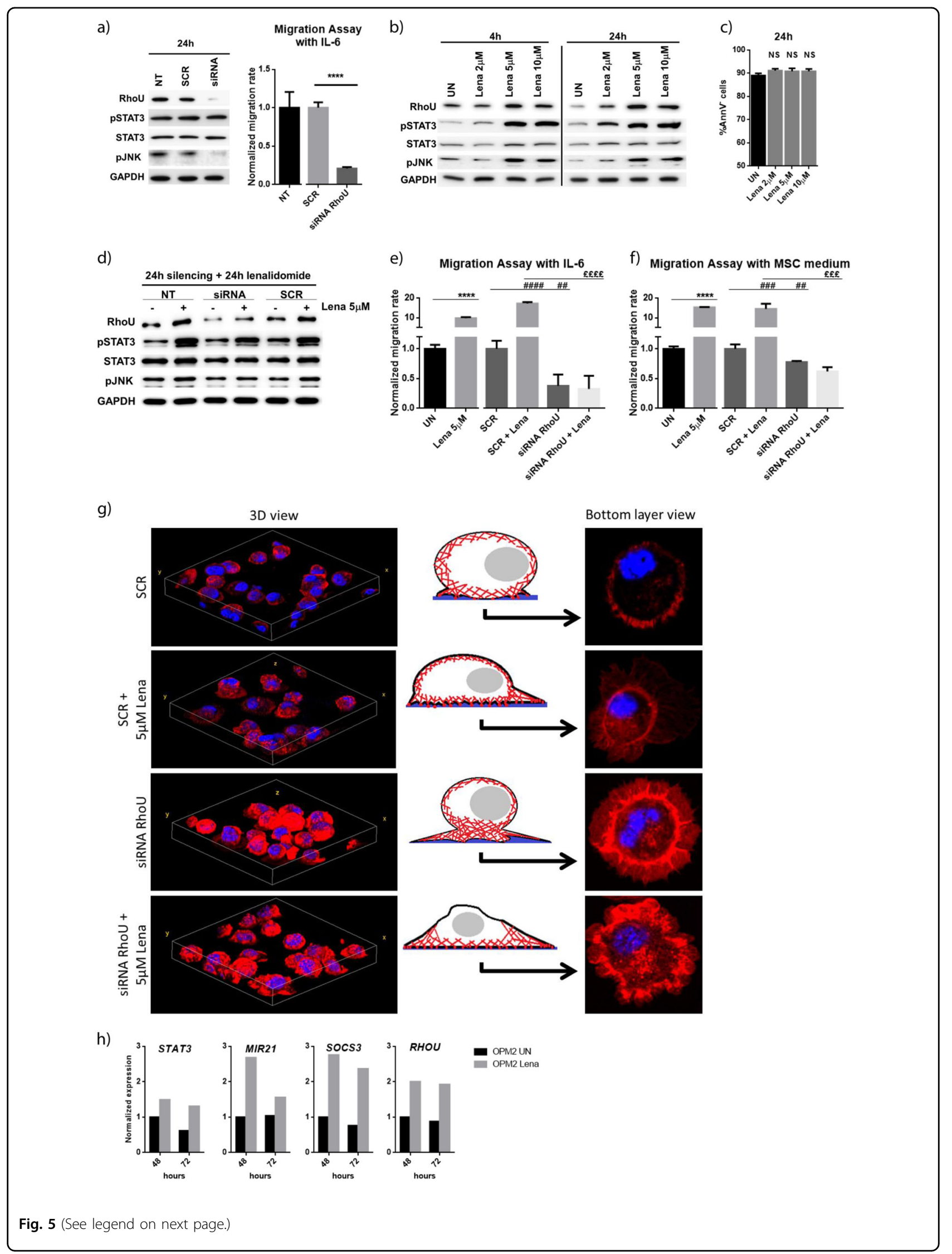




\begin{abstract}
Fig. 5 Lenalidomide enhances MM cell migration through the upregulation of RhoU. a Immunoblot of U266 proteins and bar chart exhibiting normalized mean cell count \pm SD of four samples from at least two independent experiments, after $24 \mathrm{~h}$ of transfection with SCR or RhoU siRNA. $\mathbf{b}$ Immunoblots of U266 proteins after 4 and $24 \mathrm{~h}$ of treatment with different doses of lenalidomide. $\mathbf{c}$ Bar charts showing the percentage of live cells $\left(A n n V^{-}\right.$) after treatment with different doses of lenalidomide for $24 \mathrm{~h} . \mathbf{d}$ Immunoblot of U266 proteins after $48 \mathrm{~h}$ of transfection with RhoU or SCR siRNA, lenalidomide was added in the last $24 \mathrm{~h} . \mathbf{a}, \mathbf{b}$, and $\mathbf{d}$ show representative immunoblots from six samples from at least two independent experiments; UN untreated, NT not transfected; protein expression is showed in the following order: RhoU, phospho-STAT3(Tyr705), STAT3, phosphoJNK(Thr183/Tyr185), and GAPDH. e Bar charts showing normalized mean cell count \pm SD of four samples from at least two independent experiments after $48 \mathrm{~h}$ of transfection with RhoU or SCR siRNA combined with lenalidomide treatment in the last $24 \mathrm{~h}$ accessed by migration assay with IL-6, normalized over mean migration of UN. $\mathbf{f}$ Bar charts showing normalized mean cell count \pm SD of four samples from at least two independent experiments after $48 \mathrm{~h}$ of transfection with RhoU or SCR siRNA combined with lenalidomide treatment in the last $24 \mathrm{~h}$ accessed by migration assay with MSC medium, normalized over mean migration of UN. e and $\mathbf{f} U N$ untreated ${ }^{*}$ Compared to UN; ${ }^{*}$ Compared to SCR; ${ }^{\mathbf{E}}$ Compared to SCR + Lena, ${ }^{*} p<0.05 ;{ }^{* *} p<0.01 ;{ }^{* * *} p<0.001 ;{ }^{* * *} p<0.0001$. g Changes in the actin cytoskeleton after RhoU silencing or SCR transfection combined or not with lenalidomide treatment. DAPI staining shows the nucleus in blue, phalloidin staining in red shows F-actin filaments. Cells were fixed after $48 \mathrm{~h}$ siRNA transfection and $24 \mathrm{~h}$ of $5 \mu \mathrm{M}$ lenalidomide treatment. All cells were plated at the same concentration in a six well plate for $48 \mathrm{~h}$ to maintain growth conditions. $\mathbf{h}$ Expression of STAT3 and its targets MIR21, SOCS3, and RHOU after treatment of OPM2 cell line with lenalidomide for 48 and $72 \mathrm{~h}$ (\#GSE31421)
\end{abstract}

changes in cell morphology were evident. SCR cells displayed a normal round-up morphology (3D view) with smooth edges and few spiky protrusions (bottom layer view); siRNA RhoU cells had bigger lamellipodia-like edges with an accumulation of stress fibers and focal adhesions; lenalidomide treated SCR cells were flattened (3D view) and with multiple filopodial protrusion (bottom layer view), typical of migrating cells in accordance with the migration data above; noteworthy, the combination of RhoU silencing with lenalidomide treatment resulted in a loss of cytoskeleton organization (Fig. $5 \mathrm{~g}$ ).

To deepen the study of STAT3 activation and RhoU overexpression upon lenalidomide treatment, we have analyzed the expression of STAT3 itself and its target genes MIR21, SOCS3 and RHOU in a GEP dataset of OPM2 cell line upon lenalidomide treatment for 48 and $72 \mathrm{~h}$ (\#GSE31421) $)^{50}$. Upon lenalidomide treatment there is a $50 \%$ increase in STAT3 expression, while the increase in the expression of its target genes is even more clear (Fig. 5h).

Additionally, since RhoU silencing caused cell cycle arrest we further investigated the changes in cell cycle after lenalidomide treatment or the combination of both. Twenty four-hour treatment with lenalidomide caused a significant increase in G0/G1 phase and a decrease in S phase (Fig. 6a). The combination of both RhoU silencing and lenalidomide led to a further accumulation of cells in the G0/G1 phase (Fig. 6a). We have also analyzed cyclin D2 expression, unraveling that although lenalidomide treatment impaired the transition from $\mathrm{G} 1$ to $\mathrm{S}$ phases, it did have a significant impact in cyclin D2 expression (Fig. $6 \mathrm{~b})$. However, as previously described in literature, lenalidomide treatment caused an increase in p21 expres$\operatorname{sion}^{51}$ in both conditions that might explain the impairment in cell cycle progression.

A schematic view of the proposed mechanism is depicted in Fig. 6c.

\section{Discussion}

We report here the first evidence of a clear unbalance in the expression of multiple members of the Rho GTPase family in MM PCs when compared to normal PCs. Interestingly, opposite to what was seen in other tumors where there is an over-expression of some of the members of this family ${ }^{7}$, we have found that more than $50 \%$ of Rho GTPases are actually down-modulated in MM PCs when compared to healthy PCs.

Focusing our attention on the RhoU/V family, we have found that RhoU expression is significantly modulated during the different steps of MM progression. MGUS patients showed higher RhoU levels when compared to normal controls, raising the hypothesis that this protein might be important especially in the early stages of MM malignancy. With disease progression and accumulation of malignant PCs in the BM, we observed a decrease in RHOU when compared to MGUS patients. It has been reported that high RhoU levels lead to enhanced cell motility, while low RhoU levels are essential for adhe$\operatorname{sion}^{47}$. It is predictable that RhoU is expressed at high levels in the initial steps of the disease when PCs could be more motile since they are competing for $\mathrm{BM}$ niches ${ }^{52}$. With disease progression inside the BM, cells adhere and rely on the microenvironment for their survival, which seems to translate in a cell-contact dependent decrease in RHOU expression, hypothesis supported by the in vitro evidence of a RHOU down-modulation in MM cell lines cultured in contact with HS-5 stromal cells.

Remarkably, MM patients in high risk TC groups have significantly higher levels of $R H O U$ mRNA that positively correlated with the presence of unfavorable cytogenetic alterations; however the sole presence of 1q gain, where RHOU gene is located, was not enough to cause a significant change in its expression. Also, higher levels of RHOU expression correlated with increased CCND2 and decreased cell cycle control and DNA damage repair 
a)

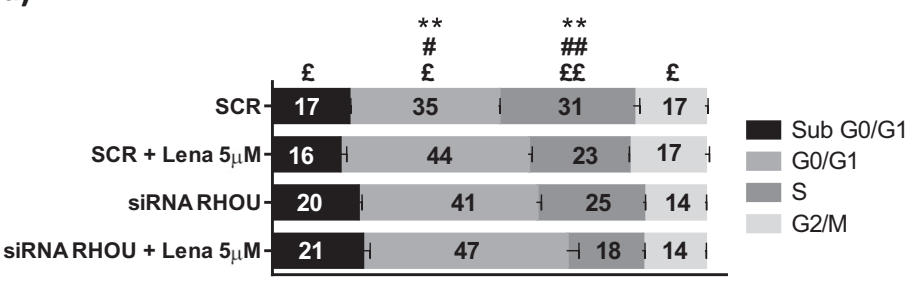

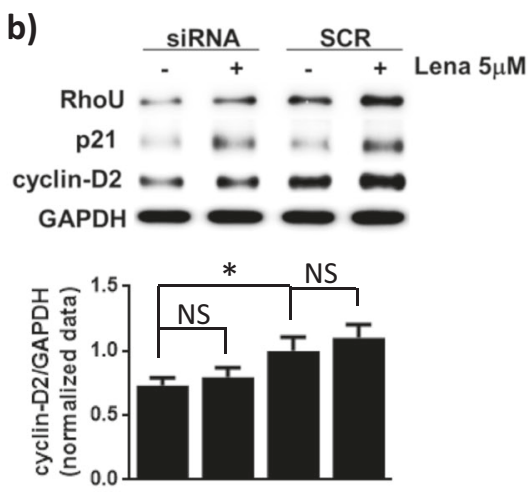

c)
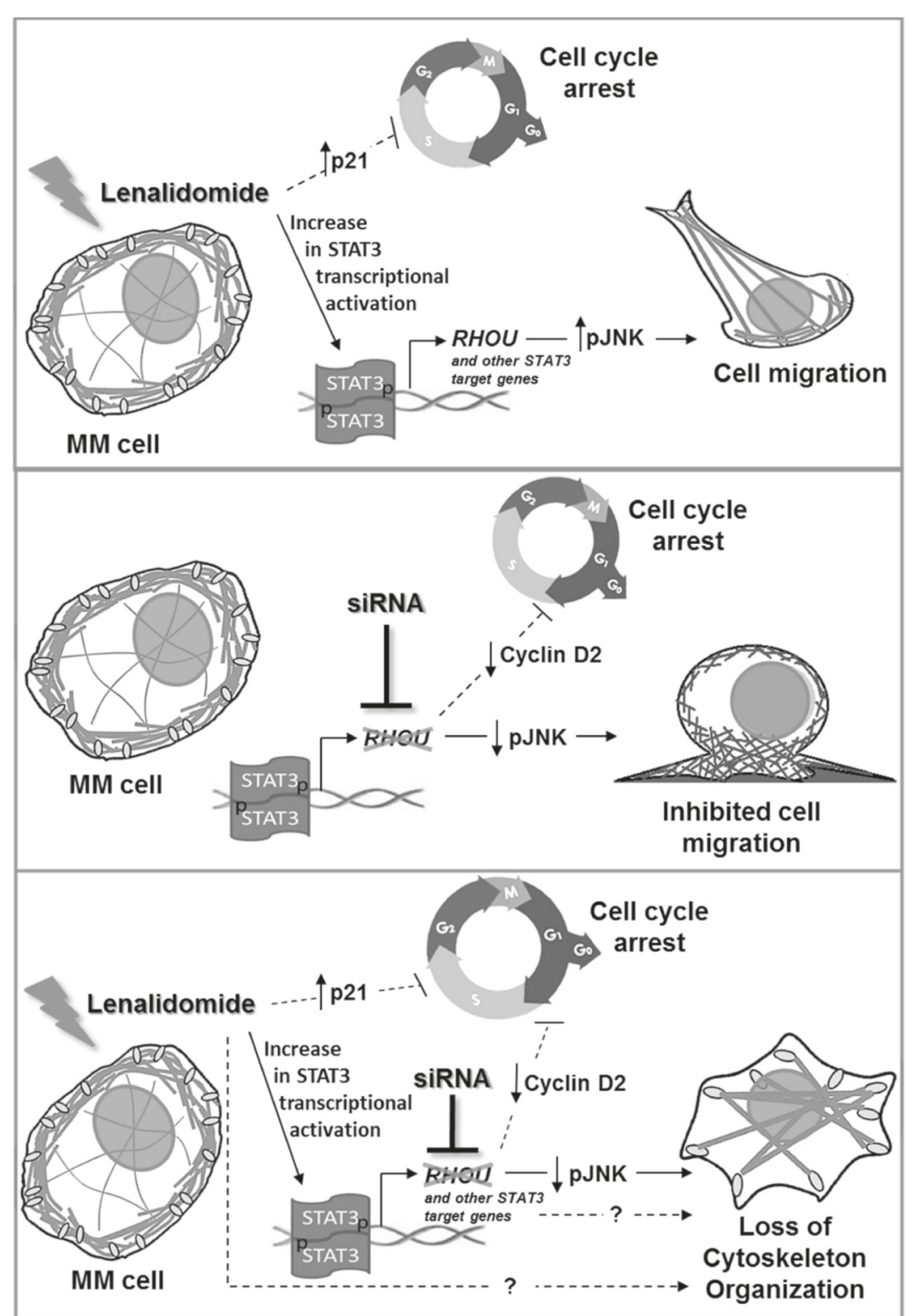

Fig. 6 (See legend on next page.) 
Fig. 6 Cell cycle analysis and schematic view of the effect of lenalidomide, RhoU silencing or their combination in MM cells. a Cell cycle analysis based on PI staining of SCR and siRNA RHOU cells $48 \mathrm{~h}$ after transfection with the addition of lenalidomide in the last $24 \mathrm{~h}$. Data represents mean \pm SD of three independent experiments. Student's $t$ test: ${ }^{*} S C R$ compared to SCR + Lena; ${ }^{*} S i R N A$ RhoU compared to siRNA RhoU + Lena; ${ }^{\ddagger}$ SiRNA RhoU + Lena compared to SCR + Lena, ${ }^{*} p<0.05 ;{ }^{* *} p<0.01$. b Representative immunoblots from six samples from at least two independent experiments of RhoU, cyclin D2, and p21 expression after siRNA transfection and lenalidomide treatments, and densitometry of the expression of cyclin D2/GAPDH. c Lenalidomide treatment determines an increase in STAT3 activation and consequently the increased expression of STAT3 target genes including RhoU. This, combined with increased levels of pJNK, results in a reorganization of the cytoskeleton and boosts cell migration. These effects are parallel to cell cycle arrest, through a mechanism that is not explored in this manuscript. RhoU inhibition, on the other hand, results in decreased levels of active JNK leading to decreased cell migration rates and increased lamellipodial protrusions. RhoU silencing also caused MM cell cycle arrest due to a decrease in the expression of cyclin D2. The combination of both RhoU silencing and lenalidomide treatment further inhibits cell cycle progression and overcomes lenalidomide induced migration by decreasing the levels of active JNK causing however a loss of cytoskeleton organization. This cytoskeleton organization loss may be due to the expression of other STAT3 target genes or due to lenalidomide targets not explored in this article

genes; while RhoU silencing resulted in a decrease in cyclin D2 protein expression and impaired cell cycle progression. Put together, these results suggest that even though most MM patients down-modulate $R H O U$ with disease progression, a high expression of this GTPase in late stages of this malignancy could actually be associated with a worse prognosis. We also found that IL-6 stimulus, important for MM survival, resulted in a STAT3dependent increase in $R H O U$ expression in cell lines. Likewise, CD45 ${ }^{+}$U266 cells, that have increased JAK/

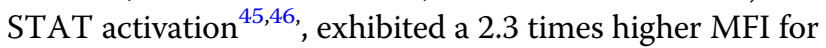
intracytoplasmic RhoU staining. To note, in MM patients RHOU expression correlated with the expression of STAT3 itself, of MIR21 and of SOCS3, confirming that it might be highly dependent on the activation of the STAT3 cascade, as observed in MM cell lines.

RhoU silencing led to a loss of migration capability probably due to the decrease in active JNK, as previous studies suggested: a constitutively active RhoU mutant was able to increase JNK activation in mouse epithelial cells $^{53}$ and RhoU depletion in HelaS3 cells inhibited JNK activating phosphorylation consequently impairing filopodium formation, a type of cell protrusion essential for cell migration ${ }^{54}$. Lastly, we described the effects of IMIDs on the IL-6/STAT3/RhoU/JNK branch. This new generation drugs for MM treatment have been shown to reorganize cells' cytoskeleton by modulating Rho GTPases in human monocytes ${ }^{49}$. Indeed, we found that lenalidomide commanded an increased activation of STAT3, augmented RhoU expression and amplified JNK phosphorylation. Parallel to the changes in cytoskeleton conformation after lenalidomide, we also observed a decrease in cell cycle progression, probably through a p21 dependent mechanism, in line with what is already known for this drug ${ }^{51,55,56}$. Consistently and opposite to what was observed with RhoU silencing, lenalidomide led to a high increase in cell motility. The combination of RhoU silencing with lenalidomide treatment led to a loss of cytoskeleton organization that translated in a defective migratory capability, and a further increase in cell cycle arrest. These results give insights into a novel mechanism of action of lenalidomide and prove that this drug can somehow stimulate the activation of STAT3 transcription factor enhancing the expression of its target genes including RHOU, leading to cytoskeletal changes in MM PCs. The increased cell motility upon lenalidomide is consistent with the disruption of microenvironment niche previously described ${ }^{57,58,}$ where less adherent cells are more easily targeted by immune cells or combined chemotherapeutics. Nevertheless, whether or not high RhoU levels indicate an increased propensity towards dissemination of malignant PCs, whether RhoU levels during lenalidomide therapy in MM patients are increased, and whether RhoU high-expressing MM could display different responsiveness to lenalidomide remains to be clarified.

\section{Acknowledgements}

We thank patients and their families for donating samples. This work was supported by grants from the Associazione Italiana per la Ricerca sul Cancro (AIRC) to F.P. (IG14481; IG18387), G.S. and A.N., from the Italian Ministry of Education, University and Research (FIRB-Futuro in Ricerca 2008) to F.P. and from the University of Padova (Progetti di ricerca di Ateneo - CPDA114940) to F.P. S.M. was supported by a Fondazione Umberto Veronesi Fellowship.

\section{Author details}

${ }^{1}$ Department of Medicine, Division of Hematology, University of Padova, Padova, Italy. ${ }^{2}$ Laboratory of Normal and Malignant Hematopoiesis, Venetian Institute of Molecular Medicine, Padova, Italy. ${ }^{3}$ Hematology Unit, Fondazione IRCCS Ca' Granda Ospedale Maggiore Policlinico, Milan, Italy. ${ }^{4}$ Department of Oncology and Hemato-Oncology, University of Milano, Milano, Italy. ${ }^{5}$ Surgical Pathology and Cytopathology Unit, Department of Medicine - DIMED, University of Padova, Padova, Italy. ${ }^{6}$ Present address: Department of Medical Oncology, Dana-Farber Cancer Institute, Boston, MA, USA

\section{Author contributions}

S.C.N. conceived and designed the study, performed research, analyzed data and wrote the manuscript. M.M., M.P., E.M., M.C., L.Q.T., and S.M. helped with experiments, partly analyzed data and revised the manuscript; R.Z., F.A., A.V., and G.B. contributed with patients' samples; L.T. and G.S. contributed with patients' samples and provided funding; A.N. provided funding and critical advices during elaboration of data. F.P. supported the design of the study, provided funding, supervised research and data analysis, and revised the manuscript.

\section{Conflict of interest}

The authors declare that they have no conflict of interest. 


\section{Publisher's note}

Springer Nature remains neutral with regard to jurisdictional claims in published maps and institutional affiliations.

Supplementary Information accompanies this paper at https://doi.org/ 10.1038/s41408-018-0053-z.

Received: 6 October 2017 Revised: 19 December 2017 Accepted: 8 January 2018

Published online: 13 February 2018

\section{References}

1. Anderson, K. C. \& Carrasco, R. D. Pathogenesis of myeloma. Annu. Rev. Pathol. 6, 249-274 (2011)

2. Morgan, G. J., Walker, Ba \& Davies, F. E. The genetic architecture of multiple myeloma. Nat. Rev. Cancer 12, 335-348 (2012).

3. Landgren, $\mathrm{O}$. et al. Monoclonal gammopathy of undetermined significance (MGUS) consistently precedes multiple myeloma: a prospective study. Blood 113, 5412-5417 (2009).

4. Noonan, K. \& Borrello, I. The immune microenvironment of myeloma. Cancer Microenviron. 4, 313-323 (2011).

5. Vetter, I. R. \& Wittinghofer, A. The guanine nucleotide-binding switch in three dimensions. Science 294, 1299-1304 (2001).

6. Aspenström, P., Ruusala, A. \& Pacholsky, D. Taking Rho GTPases to the next level: the cellular functions of atypical Rho GTPases. Exp. Cell Res. 313, 3673-3679 (2007).

7. Vega, F. M. \& Ridley, A. J. Rho GTPases in cancer cell biology. FEBS Lett. 582, 2093-2101 (2008).

8. Raftopoulou, M. \& Hall, A. Cell migration: Rho GTPases lead the way. Dev. Biol. 265, 23-32 (2004).

9. Risse, S. L. et al. SH3-mediated targeting of Wrch1/RhoU by multiple adaptor proteins. Biol. Chem. 394, 421-432 (2013).

10. Dickover, M. et al. The atypical Rho GTPase, RhoU, regulates cell-adhesion molecules during cardiac morphogenesis. Dev. Biol. 389, 182-191 (2014).

11. Schiavone, D. et al. The RhoU/Wrch1 Rho GTPase gene is a common transcriptional target of both the gp130/STAT3 and Wnt-1 pathways. Biochem. J. 421, 283-292 (2009).

12. Fort, P. et al. Activity of the RhoU/Wrch1 GTPase is critical for cranial neural crest cell migration. Dev. Biol. 350, 451-463 (2011).

13. Todoerti, $\mathrm{K}$. et al. Transcriptional characterization of a prospective series of primary plasma cell leukemia revealed signatures associated with tumor progression and poorer outcome. Clin. Cancer Res. 19, 3247-3258 (2013).

14. Ronchetti, D. et al. Distinct IncRNA transcriptional fingerprints characterize progressive stages of multiple myeloma. Oncotarget 7, 14814-14830 (2016).

15. López-Corral, L. et al. Transcriptome analysis reveals molecular profiles associated with evolving steps of monoclonal gammopathies. Haematologica 99, 1365-1372 (2014).

16. Hideshima, T., Bergsagel, P. L., Kuehl, W. M. \& Anderson, K. C. Advances in biology of multiple myeloma: clinical applications. Blood 104, 607-618 (2004).

17. Agnelli, L. et al. Molecular classification of multiple myeloma: a distinct transcriptional profile characterizes patients expressing CCND1 and negative for 14q32 translocations. J. Clin. Oncol. 23, 7296-7306 (2005).

18. Agnelli, L. et al. Upregulation of translational machinery and distinct genetic subgroups characterise hyperdiploidy in multiple myeloma. Br. J. Haematol. 136, 565-573 (2007).

19. Manni, S. et al. Inactivation of CK1a in multiple myeloma empowers drug cytotoxicity by affecting AKT and $\beta$-catenin survival signaling pathways. Oncotarget 2017. doi:10.18632/oncotarget.14654.

20. Zhan, F. et al. The molecular classification of multiple myeloma. Blood $\mathbf{1 0 8}$ 2020-2028 (2006).

21. Chen, L. et al. Identification of early growth response protein 1 (EGR-1) as a novel target for JUN-induced apoptosis in multiple myeloma. Blood $\mathbf{1 1 5}$ 61-70 (2010).

22. Hanamura, I., Huang, Y., Zhan, F., Barlogie, B. \& Shaughnessy, J. Prognostic value of cyclin D2 mRNA expression in newly diagnosed multiple myeloma treated with high-dose chemotherapy and tandem autologous stem cell transplantations. Leukemia 20, 1288-1290 (2006).

23. Heuck, C. J. et al. Five gene probes carry most of the discriminatory power of the 70-gene risk model in multiple myeloma. Leukemia 28, 2410-2413 (2014).
24. Li, D., Das, S. Yamada, T. \& Samuels, H. H. The NRIF3 family of transcriptional coregulators induces rapid and profound apoptosis in breast cancer cells. Mol. Cell Biol. 24, 3838-3848 (2004).

25. Orjalo, A. V. et al. The Nup107-160 Nucleoporin complex is required for correct bipolar spindle assembly. Mol. Biol. Cell 17, 3806-3818 (2006).

26. Platani, M. et al. The Nup107-160 nucleoporin complex promotes mitotic events via control of the localization state of the chromosome passenger complex. Mol. Biol. Cell 20, 5260-5275 (2009).

27. Thomas, Y., Coux, O. \& Baldin, V. BTrCP-dependent degradation of CDC25B phosphatase at the metaphase-anaphase transition is a pre-requisite for correct mitotic exit. Cell Cycle 9, 4338-4350 (2010).

28. Osman, F. \& Whitby, M. C. Emerging roles for centromere-associated proteins in DNA repair and genetic recombination. Biochem. Soc. Trans. 41, 1726-1730 (2013).

29. Kim, H., Fonseca, C. \& Stumpff, J. A unique kinesin-8 surface loop provides specificity for chromosome alignment. Mol. Biol. Cell 25, 3319-3329 (2014).

30. Song, H., Park, J. E. \& Jang, C. Y. DDA3 targets Cep290 into the centrosome to regulate spindle positioning. Biochem. Biophys. Res. Commun. 463, 88-94 (2015).

31. Gozuacik, D. et al. Identification and functional characterization of a new member of the human Mcm protein family: hMcm8. Nucleic Acids Res. 31, 570-579 (2003)

32. Pollok, S., Stoepel, J., Bauerschmidt, C., Kremmer, E. \& Nasheuer, H.-P. Regulation of eukaryotic DNA replication at the initiation step. Biochem. Soc. Trans. 31, 266-269 (2003).

33. Henninger, E. E., Pursell, Z. F., Henninger, E. E. \& Pursell, Z. F. DNA polymerase $\varepsilon$ and its roles in genome stability. lubmb. Life 66, 339-351 (2014).

34. Wäsch, R., Robbins, Ja. \& Cross, F. R. The emerging role of APC/CCdh1 in controlling differentiation, genomic stability and tumor suppression. Oncogene 29, 1-10 (2010).

35. Hurt, E. M. et al. Overexpression of c-maf is a frequent oncogenic event in multiple myeloma that promotes proliferation and pathological interactions with bone marrow stroma. Cancer Cell 5, 191-199 (2004).

36. Boncela, J. et al. Plasminogen activator inhibitor type 1 interacts with alpha3 subunit of proteasome and modulates its activity. J. Biol. Chem. 286 6820-6831 (2011).

37. Bakkenist, C. J. \& Kastan, M. B. DNA damage activates ATM through intermolecular autophosphorylation and dimer dissociation. Nature 421, 499-506 (2003).

38. Jeong, S. -J. et al. Role of RNA polymerase ॥ carboxy terminal domain phosphorylation in DNA damage response. J. Microbiol. 43, 516-522 (2005).

39. Klungland, A. \& Bjelland, S. Oxidative damage to purines in DNA: role of mammalian Ogg1. DNA Repair 6, 481-488 (2007).

40. $\mathrm{Wu}, \mathrm{J} ., \mathrm{Lu}, \mathrm{L} . \mathrm{Y} . \& \mathrm{Yu}, \mathrm{X}$. The role of BRCA1 in DNA damage response. Protein Cell 1, 117-123 (2010).

41. Roy, N., Bagchi, S. \& Raychaudhuri, P. Damaged DNA binding protein 2 in reactive oxygen species (ROS) regulation and premature senescence. Int. J. Mol. Sci. 13, 11012-11026 (2012).

42. Eddy, S. et al. Human Rev1 polymerase disrupts G-quadruplex DNA. Nucleic Acids Res. Nucleic Acids Res. 42, 3272-3285 (2014).

43. Abdou, I., Poirier, G. C., Hendzel, M. J. \& Weinfeld, M. DNA ligase III acts as a DNA strand break sensor in the cellular orchestration of DNA strand break repair. Nucleic Acids Res. 43, 875-892 (2015).

44. Saito, Y. \& Komatsu, K. Functional role of NBS1 in radiation damage response and translesion DNA synthesis. Biomolecules 5, 1990-2002 (2015).

45. Lin, $H$. et al. An activated JAK/STAT3 pathway and CD45 expression are associated with sensitivity to Hsp90 inhibitors in multiple myeloma. Exp. Cell Res. 319, 600-611 (2013).

46. Mahmoud, M. S. \& Ishikawa, H. \& Fujii, R. \& Kawano, M. M. Induction of CD45 expression and proliferation in U-266 myeloma cell line by interleukin-6. Blood 92, 3887-3897 (1998).

47. Faure, S. \& Fort, P. Atypical RhoV and RhoU GTPases control development of the neural crest. Small GTPases 2, 310-313 (2011).

48. Schust, J., Sperl, B., Hollis, A., Mayer, T. U. \& Berg, T. Stattic: a small-molecule inhibitor of STAT3 activation and dimerization. Chem. Biol. 13, 1235-1242 (2006).

49. $\mathrm{Xu}$, Y. et al. Immunomodulatory drugs reorganize cytoskeleton by modulating Rho GTPases. Blood 114, 338-345 (2009).

50. Zhu, Y. X. et al. Cereblon expression is required for the antimyeloma activity of lenalidomide and pomalidomide. Blood 118, 4771-4779 (2011). 
51. Escoubet-Lozach, L. et al. Pomalidomide and lenalidomide induce p21WAF-1 expression in both lymphoma and multiple myeloma through a LSD1mediated epigenetic mechanism. Cancer Res. 69, 7347-7356 (2009).

52. Podar, K., Chauhan, D. \& Anderson, K. C. Bone marrow microenvironment and the identification of new targets for myeloma therapy. Leukemia 23, 10-24 (2009).

53. Tao, W., Pennica, D., Xu, L., Kalejta, R. F. \& Levine, A. J. Wrch-1, a novel member of the Rho gene family that is regulated by Wnt-1. Genes Dev. 15, 1796-1807 (2001).

54. Chuang, Y., Valster, A., Coniglio, S. J., Backer, J. M. \& Symons, M. The atypical Rho family GTPase Wrch-1 regulates focal adhesion formation and cell migration. J. Cell Sci. 120, 1927-1934 (2007).

55. Quach, H., Kalff, A. \& Spencer, A. Lenalidomide in multiple myeloma: current status and future potential. Am. J. Hematol. 87, 1089-1095 (2012).
56. Fink, E. C. \& Ebert, B. L. The novel mechanism of lenalidomide activity. Blood 126, 2366-2369 (2015)

57. Roboz, G. J. Novel approaches to the treatment of acute myeloid leukemia Hematol. Am. Soc. Hematol. Educ. Program. 2011, $43-50$ (2011).

58. Kotla, V. et al. Mechanism of action of lenalidomide in hematological malignancies. J. Hematol. Oncol. 2, 36 (2009).

59. Tubi, L. Q. et al. Protein kinase CK2 regulates AKT, NF-KB and STAT3 activation, stem cell viability and proliferation in acute myeloid leukemia. Leukemia $\mathbf{3 1}$, 292-300 (2016)

60. Tusher, V. G., Tibshirani, R. \& Chu, G. Significance analysis of microarrays applied to the ionizing radiation response. Proc. Natl Acad. Sci. USA 98, 5116-5121 (2001). 